\title{
Analysis of Asymmetric Polling Control System in Wireless Sensor Networks
}

\author{
Zhijun Yang ${ }^{\text {a,b,*}, \text { Zheng Liu }}{ }^{\mathrm{a}}$, Yangyang Sun ${ }^{\mathrm{a}}$, and Hongwei Ding ${ }^{\mathrm{a}}$ \\ ${ }^{a}$ School of Information Science and Technology, Yunnan University, Kunming, 650500, China \\ ${ }^{b}$ Educational and Scientific Institute, Educational Department of Yunnan Province, Kunming, 650223, China
}

\begin{abstract}
To solve the problem of the priorities of network traffic and improve the applicability of multi-access for communication systems, a twolevel asymmetric polling control system was proposed, in which the central node adopts an exhaustive access policy and the normal nodes adopt an asymmetric 1-limited $(K=1)$ access policy. This model can distinguish network traffic priorities as well as guarantee fairness of the system. Using a probability generating function and embedded Markov chain, the system model was established. Through the accurate mathematical analysis of the system model, the mathematical analytical formulas of important system performance parameters, such as the mean queue length of nodes and the mean delay of information packets, were obtained. The model provides high-quality services for high-priority nodes while maintaining the quality of service at low-priority nodes. Finally, the polling access control strategy was implemented by Tinyos.
\end{abstract}

Keywords: polling control system; priorities; asymmetric 1-limited $(K=1)$ service; fairness; TinyOS

(Submitted on September 16, 2019; Revised on September 29, 2019; Accepted on October 19, 2019)

(C) 2019 Totem Publisher, Inc. All rights reserved.

\section{Introduction}

The Internet of things is an important part of the new generation of information technology, and it is also another revolutionary transformation following Internet and mobile communication networks. The meaning of Internet of things includes not only the integration of objects into networks for identification and control [1-2], but also the transmission and intelligent processing of information. With the development of Internet of things technology, the network connection and information sharing of all items will be realized [3-5]. Wireless sensor networks (WSN) are multi-hop self-organizing network systems formed by a large number of sensor nodes deployed in monitoring areas and communicating with each other, and they also construct the technical basis of IoT [6-9].

The polling control system is a representation of scheduling control models with good, fair, and reasonable allocation of limited resources. It has been widely used in modern computer networks [10-13]. Papers [14] and [15] analysed the application of polling control systems in wireless sensor networks. Paper [16] studied its application in FPGA data acquisition systems.

For polling control systems, the arrival time of packets entering each node, the switching time between nodes, and the service time of each node transmitting an information packet are all random variables. When the probability distributions of random variables in all queues are the same, the system is a symmetric polling control system; otherwise, the system is an asymmetric polling control system. With the rapid development of modern network technology, the types of communication services are increasing. The same communication systems must meet the needs of various traffic asymmetric polling control systems to correspond to the practice better.

To distinguish traffic priorities, paper [17] used an exhaustive access policy for the central node and a gated access policy for the normal nodes. In paper [18], the discrete time exhaustive service two-level polling control system was

* Corresponding author.

E-mail address: $1029381131 @$ qq.com 
analysed and studied. In paper [19], the continuous time one-limited service and exhaustive service two level polling control system were analysed and studied. Although this system could distinguish traffic priorities, it assumed the system was symmetric.

In view of the above problems, this paper proposed a two-stage polling control system, in which an exhaustive access policy was adopted for the center node and an asymmetric one-limited $(\mathrm{K}=1)$ access policy was used for the normal nodes. This system can distinguish traffic priorities based on an asymmetric mechanism. Using a probability generating function and embedded Markov chains [20], the system model was accurately analysed. The correctness of the theoretical analysis and the effectiveness of the system were addressed through theoretical calculations and simulation experiments. Finally, the system was also designed and verified based on TinyOS.

\section{Asymmetric Polling Control System in WSN}

WSNs deploy a mass of wireless sensor nodes in the designated area, as shown in Figure 1, and the nodes that can sense and process environmental data use wireless connections to form networks in a multi hop and self-organized manner. The information perceived and collected is transmitted to the sink node through wireless communication, which passes data to the user through the Internet.

In order to solve the problem of energy loss caused by random multi-access, guarantee the fairness of packet transmission, and improve the applicability of a communication system's multi-access, based on the principle of this system, a two-level priority polling control strategy for wireless sensor networks is proposed. As shown in Figure 2, this model consists of a server (cluster head node), a center queue (center node in the cluster), and $c$ and $M$ normal queues (normal nodes in the cluster). The cluster head node acts as a logical control center, and it controls the access rights of the channel. The center node uses an exhaustive service strategy to transmit high-priority packets, and normal nodes deliver low-priority packets through an asymmetric one-limited $(K=1)$ service strategy. At the beginning, the center node $c$ with no packets is allowed to access the channel. Simultaneously, the cluster head node queries the normal node $n(n=1,2, \cdots, M ; n \neq c)$. Thus, when the normal node $n$ is allowed to access the channel, it does not need a query conversion time. It is allowed to deliver packets with asymmetric one-limited $(K=1)$ service strategy regulation, which only allows for the transmission of one packet in a polling cycle. When the node $n$ transmits one packet, the server goes through a query conversion time and turns to the poll central node again. After the service is completed, the normal node $n+1$ is allowed to transmit packets in order. This scheme ensures that the center node has higher priority and receives more services. The asymmetrical system is more suitable and flexible for multi-access and guarantees system fairness.

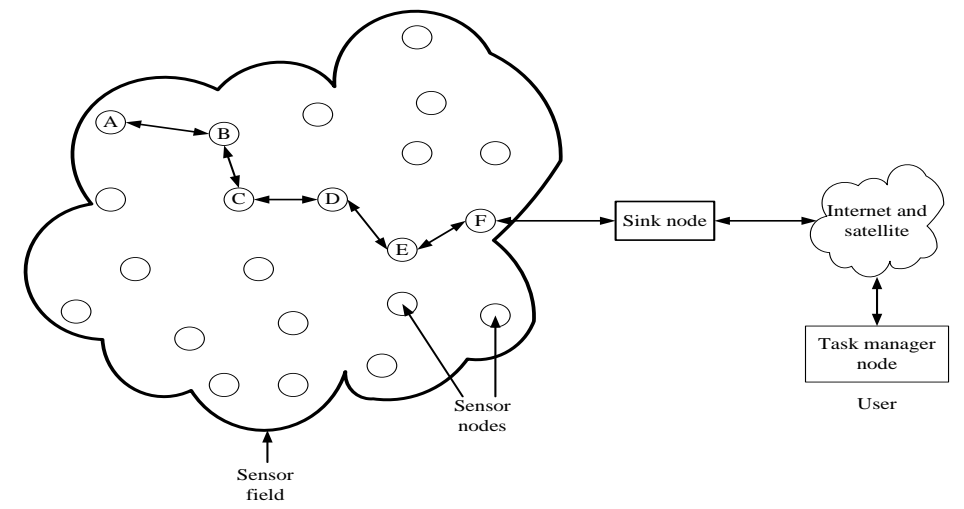

Figure 1. Architecture of wireless sensor network

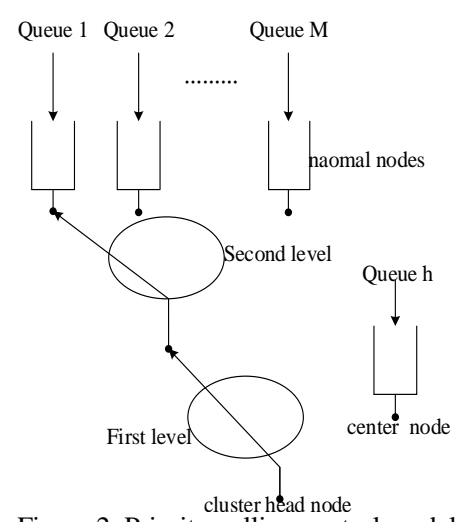

Figure 2. Priority polling control model

\section{Signal Analyses of the Theoretical Model}

Assume that the normal node $n(n=1,2, \cdots, M ; n \neq c)$ is allowed to transmit packets at $t_{m}$, the state random variable $\zeta_{n}(m)$ is the number of packets contained in the $n$ node, and the state random variable $\zeta_{c}(m)$ is the number of packets contained in the $c$ node. Then, the state random variables of the entire polling system at $t_{m}$ can be expressed as $\zeta_{1}(m), \zeta_{2}(m), \cdots, \zeta_{M}(m), \zeta_{c}(m)$. When the server begins to transmit the center node $c$ at $t_{m}^{*}$, the state random variable of center node $c$ is expressed as $\zeta_{c}^{*}(m)$, and the state random variables of the entire polling system can be expressed as $\zeta_{1}^{*}(m), \zeta_{2}^{*}(m), \cdots, \zeta_{M}^{*}(m), \zeta_{c}^{*}(m)$. Define $\zeta_{1}(m+1), \zeta_{2}(m+1), \cdots, \zeta_{M}(m+1), \zeta_{c}(m+1)$ as the state random variables of the entire polling system for the normal node $n+1$ to accept the service at $t_{m+1}$. 


\subsection{System Variables Definition}

1) The information packets arriving at each node are subject to the independent and identically distributed Poisson process, and the probabilistic generation functions, mean values, and variances of the distribution $\operatorname{are~} \mathrm{L}_{\mathrm{k}}\left(\mathrm{z}_{\mathrm{k}}\right), \lambda_{\mathrm{k}}=\mathrm{L}_{\mathrm{k}}^{\prime}(1)$, $\sigma_{\lambda \mathrm{k}}^{2}=\mathrm{L}_{\mathrm{k}}{ }^{\prime \prime}(1)+\lambda_{\mathrm{k}}-\lambda_{\mathrm{k}}^{2}, \mathrm{k}=1,2, \cdots, \mathrm{M}, \mathrm{c}$, respectively.

2) The server's service time for information packets at each node is subject to an independent and same probability distribution, and the probabilistic generation functions, mean values, and variances of the distribution are $B_{k}\left(z_{k}\right), \beta_{k}=$ $B_{k}^{\prime}(1), \sigma_{\beta k}^{2}=B_{k}{ }^{\prime \prime}(1)+\beta_{k}-\beta_{k}^{2}, k=1,2, \cdots, M, c$, respectively.

3) The switching time of the server from normal node to central node is subject to an independent and same probability distribution, and the probabilistic generation functions, mean values, and variances of the distribution are $R_{k}\left(z_{k}\right), \gamma_{k}=$ $R_{k}^{\prime}(1), \sigma_{\gamma k}^{2}=R_{k}^{\prime \prime}(1)+\gamma_{k}-\gamma_{k}^{2}, k=1,2, \cdots, M$, respectively.

4) The random variable of the time required by the central station to exhaustively service the amount of information packets arriving in any time slot is subject to a mutually independent, identically distributed probability distribution whose probability generation function is $F_{c}\left(z_{c}\right)$, where $F_{c}\left(z_{c}\right)=A_{c}\left(B_{c}\left(z_{c} F_{c}\left(z_{c}\right)\right)\right)$.

5) The buffer capacity of any queue in the service system is set to be large enough, and there is no loss of information packets. The information packets in the queue will be received according to the first-come-first-served service (FCFS).

- $u_{n}(m)$ : the switching time from the normal node $m$ to the center node $c$;

- $v_{n}(m)$ : the service time for the normal node to transmit the information packets;

- $v_{c}\left(m^{*}\right)$ : the service time for the center node to transmit the information packets;

- $\mu_{k}\left(\mu_{n}\right)$ : the number of information packets entering the node $k$ during $u_{n}(m)$ time;

- $\eta_{k}\left(v_{n}\right)$ : the number of information packets entering the node $\mathrm{k}$ during $v_{n}(m)$ time;

- $\eta_{k}\left(v_{c}\right)$ : the number of information packets entering the node $\mathrm{k}$ during $v_{c}\left(m^{*}\right)$ time.

\subsection{Generating Function}

Under the condition for the stability of the system $\sum_{n=1}^{M} \lambda_{n} \beta_{n}+\lambda_{c} \beta_{c}=\sum_{n=1}^{M} \rho_{n}+\rho_{c}<1$, the probability distribution is shown in Equation (1).

$$
\begin{aligned}
& p\left[\zeta_{n}(m)=y_{n} ; n=1,2,3, \cdots, M, c\right]=\pi_{n}\left(y_{1}, y_{2}, \cdots, y_{M}, y_{c}\right) \\
& p\left[\zeta_{n}\left(m^{*}\right)=y_{n} ; n=1,2,3, \cdots, M, c\right]=\pi_{n c}\left(y_{1}, y_{2}, \cdots, y_{M}, y_{c}\right)
\end{aligned}
$$

From Equation (1), the probability generating function of $\pi_{n}\left(y_{1}, y_{2}, \cdots, y_{M}\right)$ can be expressed as Equation (2).

$$
G_{n}\left(z_{1}, z_{2}, \cdots, z_{n}, \cdots, z_{M}\right)=\sum_{y_{1}=0}^{\infty} \sum_{y_{2}=0}^{\infty} \cdots \sum_{y_{i}=0}^{\infty} \cdots \sum_{y_{M}=0}^{\infty} \pi_{n}\left(y_{1}, y_{2}, \cdots, y_{M}\right) \cdot z_{1}^{y_{1}} z_{2}^{y_{2}} \cdots z_{n}^{y_{n}} \cdots z_{M}^{y_{M}}
$$

According to Equation (2), the probability generating function of state variables of the system when the polling queue of $n$ is at time $t_{m}$ is given by Equation (3).

$$
G_{n}\left(z_{1}, z_{2}, \cdots, z_{M}, z_{c}\right)=\lim _{t \rightarrow \infty} E\left[\prod_{n=1}^{M} z_{n}^{\zeta_{n}(m)} z_{c}^{\zeta_{c}(m)}\right]
$$

It is known from the working principle of the system that when the polling queue of $c$ is at time $t_{m}^{*}$ and the polling center queue of $n+1$ is at time $t_{m+1}$, there are the following relationships:

$$
\left\{\begin{array}{l}
\zeta_{l}\left(m^{*}\right)=\zeta_{l}(m)+\mu_{l}\left(\mu_{n}\right)+\eta_{l}\left(v_{n}\right), l=1,2, \cdots, M, c ; l \neq n \\
\zeta_{n}\left(m^{*}\right)=\mu_{l}\left(\mu_{n}\right)+\eta_{n}\left(v_{n}\right) \\
\zeta_{l}(m+1)=\zeta_{l}\left(m^{*}\right)+\zeta_{l}\left(v_{c}\right), l=1,2, \cdots, M, c \\
\zeta_{c}(m+1)=0
\end{array}\right.
$$


When the polling center queue of $c$ is at time $t_{m}^{*}$, the probability generating function of state random variables of the entire polling system is shown in Equation (5).

$$
G_{n c}\left(z_{1}, z_{2}, \cdots, z_{M}, z_{c}\right)=\lim _{t \rightarrow \infty} E\left[\prod_{n=1}^{M} z_{n}^{\zeta_{n}\left(m^{*}\right)} z_{c}^{\zeta_{c}\left(m^{*}\right)}\right]
$$

When the polling center queue of $n+1$ is at time $t_{m+1}$, the probability generating function of state random variables of the entire polling system is expressed as Equation (6).

$$
G_{n c}\left(z_{1}, z_{2}, \cdots, z_{M}, z_{c}\right)=\lim _{t \rightarrow \infty} E\left[\prod_{n=1}^{M} z_{n}^{\zeta_{n}(m+1)} z_{c}^{\zeta_{c}(m+1)}\right]
$$

From Equation (3) to (5), we obtain

$$
\begin{gathered}
G_{n c}\left(z_{1}, z_{2}, \cdots, z_{M}, z_{c}\right)=R_{n}\left(L_{c}\left(z_{c}\right) \prod_{j=1}^{M} L_{j}\left(z_{j}\right)\right) \\
\left(\frac{1}{z_{c}} B_{n}\left(L_{n}\left(z_{n}\right) \prod_{j=1}^{M} L_{j}\left(z_{j}\right)\right)\left[G_{n}\left(z_{1}, z_{2}, \cdots z_{M}, z_{c}\right)-G_{n}\left(z_{n}, \cdots, z_{n-1}, 0, z_{n+1}\right)\right]+G_{n}\left(z_{n}, \cdots, z_{n-1}, 0, z_{n+1}\right)\right)
\end{gathered}
$$

From Equation (3), (4), and (6), we obtain

$$
G_{\mathrm{n}+1}\left(z_{1}, z_{2}, \cdots, z_{M}, z_{c}\right)=G_{n c}\left(z_{1}, z_{2}, \cdots, z_{M}, B_{c}\left(\prod_{j=1}^{M} L_{j}\left(z_{j}\right) F_{c}\left(\prod_{j=1}^{M} L_{j}\left(z_{j}\right)\right)\right)\right)
$$

$F_{c}\left(z_{c}\right)=E\left[z_{c}^{F_{c}}\right]$ denotes the probability generating function $F_{c}\left(z_{c}\right)$, from which a functional equation has been derived in [21] as $F_{c}\left(z_{c}\right)=L_{c}\left(B_{c}\left(z_{c} F_{c}\left(z_{c}\right)\right)\right)$.

\section{System Characteristics}

\subsection{Mean Queue Length}

When queue $n$ starts to transmit information packets at time $t_{m}$, the number of information packets is expressed as $g_{n}(j)$, which is defined as the mean queue length.

$$
g_{n}(j)=\lim _{z_{1}, z_{2}, \cdots, z_{M} \rightarrow 1} \frac{\partial G_{n}\left(z_{1}, z_{2}, \cdots, z_{M}\right)}{\partial z_{n}}
$$

According to Equation (9) and the derivatives of Equations (7) and (8), the mean queue length of the center queue $c$ can be obtained.

$$
g_{n c}(c)=\frac{\lambda_{c} \gamma_{n}\left(1-\rho_{c}\right)}{1-\rho_{c}-\sum_{n=1}^{M} \rho_{n}}
$$

\subsection{Mean Cycle Time}

The period between two polls for one queue is expressed as $\theta$, which is defined as the mean cycle time. According to Equations (7) and (8), $\theta$ can be expressed as

$$
\theta=\frac{\sum_{n=1}^{M} \gamma_{n}}{1-\rho_{c}-\sum_{n=1}^{M} \rho_{n}}
$$




\subsection{System Throughput}

The number of information packets that the system can serve in a unit time is expressed as $T$, which is defined as the system throughput.

$$
T=\sum_{j=1}^{M} \lambda_{j} \beta_{j}+\lambda_{c} \beta_{c}
$$

\subsection{Mean Waiting Time}

The second derivative of the system is defined as

$$
\begin{gathered}
g_{n}(x, y)=\lim _{z_{1}, z_{2}, \cdots, z_{j}, \cdots, z_{M}, z_{c} \rightarrow 1} \frac{\partial^{2} G_{n}\left(z_{1}, z_{2}, \cdots, z_{n}, \cdots, z_{M}, z_{c}\right)}{\partial z_{x} \partial z_{y}} \\
g_{n c}(x, y)=\lim _{z_{1}, z_{2}, \cdots, z_{j}, \cdots, z_{M}, z_{c} \rightarrow 1} \frac{\partial^{2} G_{n c}\left(z_{1}, z_{2}, \cdots, z_{n}, \cdots, z_{M}, z_{c}\right)}{\partial z_{x} \partial z_{y}}
\end{gathered}
$$

According to Equations (13) and (14) and the derivatives of Equations (7) and (8), we obtain

$$
\begin{aligned}
& g_{n c}(c, c)=R_{n}^{\prime \prime}(1) \lambda_{c}^{2}+\gamma_{n} L_{c}^{\prime \prime}(1)+\left[2 \gamma_{n} \lambda_{c}^{2} \beta_{n}+B_{n}^{\prime \prime}(1) \lambda_{c}^{2}+\beta_{n} L_{c}^{\prime \prime}(1)\right] \theta-2 \lambda_{c} \beta_{n} g_{n 0}(c) \\
& g_{n c}(c, k)=R_{n}^{\prime \prime}(1) \lambda_{k} \lambda_{c}+\gamma_{n} \lambda_{k} \lambda_{c}+\left[2 \gamma_{n} \beta_{n} \lambda_{c} \lambda_{k}+B_{n}^{\prime \prime}(1) \lambda_{k} \lambda_{c}+\beta_{n} \lambda_{k} \lambda_{c}\right] \theta+\lambda_{c}\left[\gamma_{n}+\beta_{n}\right] g_{n}(k) \\
& -\beta_{n}\left(\lambda_{c} g_{n 0}(k)+\lambda_{k} g_{n 0}(c)\right) \\
& g_{n c}(n, c)=R_{n}^{\prime \prime}(1) \lambda_{c} \lambda_{n}+\gamma_{n} \lambda_{c} \lambda_{n}+\left[2 \gamma_{n} \beta_{n} \lambda_{n} \lambda_{c}-\gamma_{n} \lambda_{c}-\beta_{n} \lambda_{c}+B_{n}^{\prime \prime}(1) \lambda_{c} \lambda_{n}+\lambda_{c} \rho_{n}\right] \theta \\
& +\lambda_{c}\left(\gamma_{n}+\beta_{n}\right) g_{n}(n)+\left(1-\rho_{n}\right) g_{n 0}(c) \\
& g_{n c}(n, k)=R_{n}^{\prime \prime}(1) \lambda_{k} \lambda_{n}+\gamma_{n} \lambda_{k} \lambda_{n}+\lambda_{k}\left[2 \gamma_{n} \rho_{n}-\gamma_{n}-\beta_{n}+B_{n}^{\prime \prime}(1) \lambda_{n}+\rho_{n}\right] \theta+\lambda_{n}\left(\gamma_{n}+\beta_{n}\right) g_{n}(k) \\
& +\left(1-\rho_{n}\right) g_{n 0}(k)+g_{n}(n, k) \\
& \begin{array}{c}
g_{n c}(n, n)=R_{n}^{\prime \prime}(1) \lambda_{n}^{2}+\gamma_{n} L_{n}^{\prime \prime}(1)+\left[2 \gamma_{n} \beta_{n} \lambda_{n}^{2}-2 \lambda_{n} \gamma_{n}+2-2 \rho_{n}+B_{n}^{\prime \prime}(1) \lambda_{n}^{2}+\beta_{n} L_{n}^{\prime \prime}(1)\right] \theta \\
+\left(2 \lambda_{n} \gamma_{n}+2 \rho_{n}-2\right) g_{n}(n)+g_{n}(n, n)
\end{array} \\
& g_{n c}(k, l)=R_{n}^{\prime \prime}(1) \lambda_{k} \lambda_{l}+\gamma_{n} \lambda_{k} \lambda_{l}+\lambda_{k} \lambda_{l}\left[2 \gamma_{n} \beta_{n}+B_{n}^{\prime \prime}+\beta_{n}\right] \theta+\lambda_{k}\left(\gamma_{n}+\beta_{n}\right) g_{n}(l) \\
& +\lambda_{l}\left(\gamma_{n}+\beta_{n}\right) g_{n}(k)+g_{n}(k, l) \\
& g_{n c}(k, k)=R_{n}^{\prime \prime}(1) \lambda_{k}^{2}+\gamma_{n} L_{k}^{\prime \prime}(1)+\left[2 \gamma_{n} \beta_{n} \lambda_{k}^{2}+B_{n}^{\prime \prime}(1) \lambda_{k}^{2}+\beta_{n} L_{k}^{\prime \prime}(1)\right] \theta+2 \lambda_{k}\left(\gamma_{n}+\beta_{n}\right) g_{n}(k) \\
& -2 \beta_{n} \lambda_{k} g_{n 0}(k)+g_{n}(k, k) \\
& g_{n+1}(k, n)=g_{n c}(k, n)+\lambda_{n} \beta_{c}\left(1+F_{c}^{\prime}\right) g_{n c}(k, c)+\lambda_{k} \beta_{c}\left(1+F_{c}^{\prime}\right) g_{n c}(c, n)+\lambda_{n} \lambda_{k} \beta_{c}^{2}\left(1+F_{c}^{\prime}\right)^{2} g_{n c}(c, c) \\
& +\lambda_{n} \lambda_{k} B_{c}^{\prime \prime}\left[1+F_{c}^{\prime}\right]^{2} g_{n c}(c)+\lambda_{n} \lambda_{k} \beta_{c}\left[1+3 F_{c}^{\prime}+F_{c}^{\prime \prime}\right] g_{n c}(c) \\
& g_{n+1}(k, l)=g_{n c}(k, l)+\lambda_{l} \beta_{n}\left(1+F_{n}^{\prime}\right) g_{n c}(k, c)+\lambda_{k} \beta_{c}\left(1+F_{c}^{\prime}\right) g_{n c}(c, l)+\lambda_{l} \lambda_{k} \beta_{c}^{2}\left(1+F_{c}^{\prime}\right)^{2} g_{n c}(c, c) \\
& +\lambda_{l} \lambda_{k} B_{c}^{\prime \prime}\left[1+F_{c}^{\prime}\right]^{2} g_{n c}(c)+\lambda_{l} \lambda_{k} \beta_{c}\left[1+3 F_{c}^{\prime}+F_{c}^{\prime \prime}\right] g_{n c}(c) \\
& g_{n+1}(k, k)=g_{n c}(k, k)+\lambda_{k} \beta_{c}\left(1+F_{c}^{\prime}\right) g_{n c}(k, c)+\lambda_{k} \beta_{c}\left(1+F_{c}^{\prime}\right) g_{n c}(c, k)+\lambda_{k}^{2} \beta_{c}^{2}\left(1+F_{c}^{\prime}\right)^{2} g_{n c}(c, c) \\
& +\lambda_{k}^{2} B_{c}^{\prime \prime}\left[1+F_{c}^{\prime}\right]^{2} g_{n c}(c)+\beta_{c}\left[L_{k}^{\prime \prime}+2 F_{c}^{\prime} \lambda_{k}^{2}+F_{c}^{\prime \prime} \lambda_{k}^{2}+L_{k}^{\prime \prime} F_{c}^{\prime}\right] g_{n c}(c) \\
& g_{n+1}(n, n)=g_{n c}(n, n)+\lambda_{n} \beta_{c}\left(1+F_{c}^{\prime}\right) g_{n c}(n, c)+\lambda_{n} \beta_{c}\left(1+F_{c}^{\prime}\right) g_{n c}(c, n)+\lambda_{n}^{2} \beta_{c}^{2}\left(1+F_{c}^{\prime}\right)^{2} g_{n c}(c, c) \\
& +\lambda_{n}^{2} B_{c}^{\prime \prime}(1)\left[1+F_{c}^{\prime}\right]^{2} g_{n c}(c)+\beta_{c}\left[L_{n}^{\prime \prime}+2 F_{c}^{\prime} \lambda_{n}^{2}+F_{c}^{\prime \prime}(1) \lambda_{n}^{2}+L_{n}^{\prime \prime} F_{c}^{\prime}\right] g_{n c}(c) \\
& \left\{\begin{array}{l}
g_{n+1}(n, c)=0 \\
g_{n+1}(k, c)=0
\end{array}\right.
\end{aligned}
$$


After calculating Equations (22) and (23), we obtain $\sum_{n=1}^{M} g_{n+1}(k, l)$. From the calculation of Equations (18) and (20), we obtain $\sum_{n=1}^{M} g_{n c}(k, l)$. After calculating Equations (19), (21), (24), and (25), we get $\sum_{n=1}^{M} g_{n+1}(k, k)$ and $\sum_{n=1}^{M} g_{n c}(k, k)$. Then, by calculating and simplifying Equations (15) to (17) and (26), as well as $\sum_{n=1}^{M} g_{n+1}(k, l)$, $\sum_{n=1}^{M} g_{n c}(k, l), \sum_{n=1}^{M} g_{n+1}(k, k)$, and $\sum_{n=1}^{M} g_{n c}(k, k)$, we obtain

$$
\begin{gathered}
\left\{\left(1-4 \sum \rho_{n}^{2}\right) \rho_{c}\left[1+F_{c}^{\prime}\right] \sum \rho_{n}\right\} \sum \frac{\left(1-\theta \lambda_{n}\right) \beta_{n}}{\lambda_{n}} g_{n}(n)=\frac{\sum \rho_{n} \sum R_{n}^{\prime \prime} \sum B_{n}^{\prime \prime}}{\left(1+\rho_{c}\right)^{2}}+\theta \frac{\sum \rho_{n} \sum \gamma_{n} \sum \beta_{n}}{\left(1-\rho_{c}\right)^{2}}+ \\
\frac{\sum \rho_{n} \sum \beta_{n}}{\left(1-\rho_{c}-\sum \beta_{n}\right)^{2}} \sum\left[4 \beta_{n} \gamma_{n}+A_{n}^{\prime \prime}(1)+\beta_{n}\right] \frac{\sum \beta_{n}}{1-\rho_{c}-\sum \rho_{n}}+\theta \frac{\left(1+\rho_{c}\right)^{2}}{1-\rho_{c}-\sum \rho_{n}}+ \\
\left.\frac{\sum \rho_{n} \sum \gamma_{n}^{2}\left(\sum \beta_{n}\right)^{2}}{\left(1-\rho_{c}\right)^{2}}+\frac{\sum \sum \gamma_{n}^{2}}{\left(1-\rho_{c}-\sum \rho_{n}\right)}\right]+\frac{\lambda_{c} \sum \rho_{n} \sum \gamma_{n} \sum \beta_{n}}{\left(1-\rho_{c}\right)^{2}\left(1-\rho_{c}-\sum \rho_{n}\right)}+\sum \gamma_{n} \sum \beta_{n}\left[\sum \rho_{n} \sum \gamma_{n}+\right. \\
\left.\frac{\rho_{c} \sum \beta_{n}}{\left(1-\rho_{c}-\sum \rho_{n}\right)}-\frac{\sum \sum \gamma_{n}^{2}}{\left(1-\rho_{c}\right)^{2}}\right]+\frac{\lambda_{c}^{2} \rho_{c} \sum \gamma_{n}}{1-\rho_{c}-\left(\sum \rho_{m}\right)^{2}}\left[\frac{12 \rho_{c}}{1-\rho_{c}-\sum \rho_{n}}-\frac{\lambda_{c} L_{c}^{\prime \prime} \beta_{c}^{2}}{\left(1-\rho_{c}\right)^{3}}+\right. \\
\left.\left.\frac{\lambda_{c} B_{c}^{\prime \prime}}{\left(1-\rho_{c}\right)^{3}}+\frac{5 \rho_{c}^{2}\left(\sum \rho_{n}\right)^{2}}{\left(1-\rho_{c}\right)^{2}}\right]+\frac{\left(1-\sum \beta_{n}\right) \sum \rho_{n}^{2}}{1-\sum \beta_{n}^{2} L_{n}^{\prime \prime}}+\frac{\left(1+\sum \gamma_{n}\right)\left(\sum \rho_{n}\right)^{2}}{\left(1-\rho_{c}\right)^{2}}\right]
\end{gathered}
$$

Where $\rho_{n}=\lambda_{n} \beta_{n}, \rho_{c}=\lambda_{c} \beta_{c}, \sum \rho_{n}=\sum_{n=1}^{M} \lambda_{n} \beta_{n}$.

Define two variables $\theta_{1 n}\left(z_{n}\right)$ and $\theta_{2 n}\left(z_{n}\right)$ related to the cycle period. Let them meet the requirements of the following equations:

$$
\begin{gathered}
G_{n}\left(1,1, \cdots, z_{n}, \cdots, 1\right)=\theta_{1 n}\left(z_{n}\right)\left\{\frac{B_{n}\left(L_{n}\left(z_{n}\right)\right)}{z_{n}}\left[G_{n}\left(1, \cdots, z_{n}, \cdots, 1\right)-G_{n}(0)+G_{n}(0)\right]\right. \\
\theta_{2 n}\left(z_{n}\right)=\theta_{1 n}\left(z_{n}\right)\left\{B_{n}\left(z_{n}\right)\left[1-G_{n}(0)\right]+G_{n}(0)\right\}
\end{gathered}
$$

Where $G_{n}(0)=G_{n}(1, \cdots, 0, \cdots, 1)$.

Calculating the second derivatives of Equation (28) and (29), we obtain:

$$
2\left(1-\rho_{n}\right)\left(1-\theta \lambda_{n}\right) g_{n}(n)=\lambda_{n}^{2} \theta_{2 n}^{\prime \prime}(1)+\theta L_{n}^{\prime \prime}(1)+2 \theta \lambda_{n}\left(1-\rho_{n}\right)\left(1-\theta \lambda_{n}\right)
$$

From the first derivative and second derivative of the variable $\theta_{2 n}\left(z_{n}\right)$, we obtain

$$
\theta_{2 n}^{\prime}(1)=\theta_{2 j}^{\prime}(1)=\theta, \theta_{2 n}^{\prime \prime}(1) \approx \theta_{2 j}^{\prime \prime}(1)
$$

Calculating Equations (30) and (31), we obtain

$$
\begin{aligned}
g_{n}(n)= & \frac{\lambda_{n}^{2}}{2\left(1-\rho_{n}\right)\left(1-\theta \lambda_{n}\right)} \sum_{j=1}^{M} \frac{2\left(1-\rho_{j}\right)}{\rho_{j}} \sum_{j=1}^{M} \frac{\left(1-\theta \lambda_{j}\right) \beta_{j}}{\lambda_{j}} g_{j}(j)- \\
& \frac{\lambda_{n}^{2} \theta}{2\left(1-\rho_{n}\right)\left(1-\theta \lambda_{n}\right)} \sum_{j=1}^{M} \frac{L_{j}^{\prime \prime}+2 \lambda_{j}\left(1-\rho_{j}\right)\left(1-\theta \lambda_{j}\right)}{\lambda_{j}^{2}}
\end{aligned}
$$

The mean queue length of the normal queue $g_{n}(n)$ is obtained by calculating Equations (27) and (32). Therefore, the mean queue length of the normal queue $g_{n}(n)$ and the mean queue length of the center queue $g_{n c}(c)$ have been obtained.

The delay (waiting time) of information packets refers to the period of an information packet from arriving at the queue to being served. Define $E\left(w_{n}\right)$ and $E\left(w_{c}\right)$ as the mean delays of the normal queue $n$ and the center queue $c$, respectively.

The mean delay of normal queue $m$ is

$$
E\left(w_{n}\right)=\frac{g_{n}(n)}{\theta \lambda_{n}^{2}}-\frac{1}{\lambda_{n}}-\frac{L_{n}^{\prime \prime}}{2 \lambda_{n}^{2}}
$$


The mean delay of center queue $c$ is

$$
E\left(w_{c}\right)=\frac{g_{n c}(c, c)}{2 \lambda_{c} g_{n c}(c)}-\frac{\left(1-2 \rho_{c}\right) L_{c}^{\prime \prime}}{2 \lambda_{c}^{2}\left(1-\rho_{c}\right)}+\frac{\lambda_{c} B_{c}^{\prime \prime}}{2\left(1-\rho_{c}\right)}
$$

\section{Simulation Experiment}

According to the two-level asymmetric polling control system model that was established above, simulation experiments were carried out under the condition of system stability of $\sum_{n=1}^{M} \lambda_{n} \beta_{n}+\lambda_{c} \beta_{c}=\sum_{n=1}^{M} \rho_{n}+\rho_{c}<1$. Simulation experiments were completed in the MATLAB2014a platform. The server's service time for information packets at each node obeys an exponential distribution, and the mean is expressed as $\beta_{n}$ in the normal node $n$ and $\beta_{c}$ in the center node $c$. The arrival process is a Poisson process. $\lambda_{n}$ expresses the rate of the normal node $n$, and $\lambda_{c}$ expresses the rate of the center node $c$. The switching times are independent of each other, with $\gamma_{i}$ in the center node $c$. According to Equations (10) to (12), (33), and (34), the theoretical values can be obtained. The simulation parameters of Figures 3 and 4 are listed in Table 1, the simulation parameters of Figure 5 are listed in Table 2, and the simulation parameters of Figure 6 are listed in Table 3.

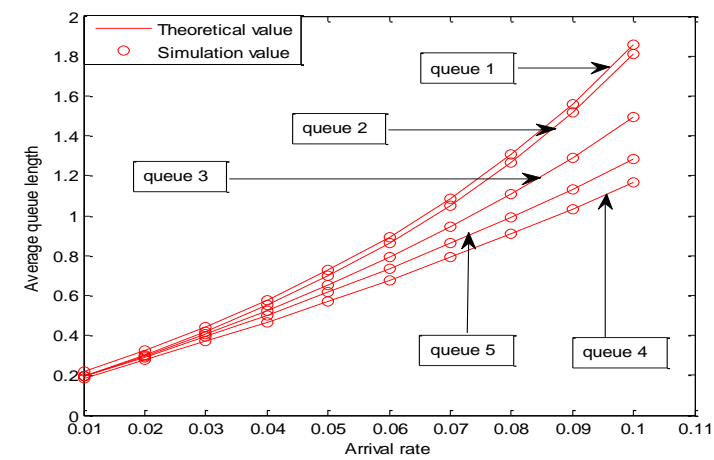

(a) Mean queue length of normal queue

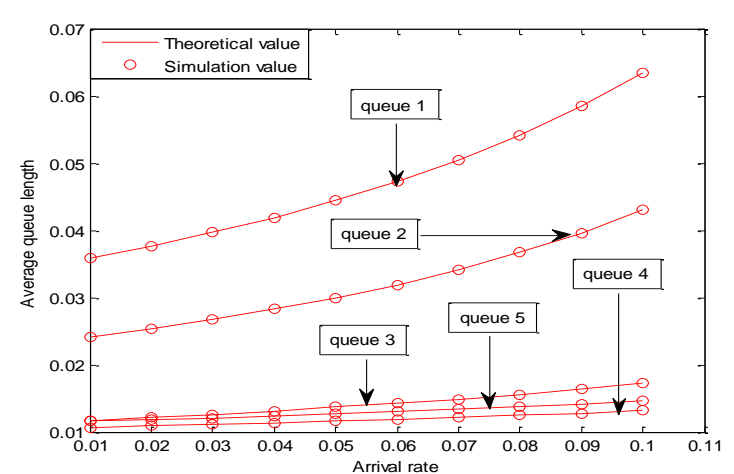

(b) Mean queue length of center queue Figure 3. The impact of arrival rate on mean queue length

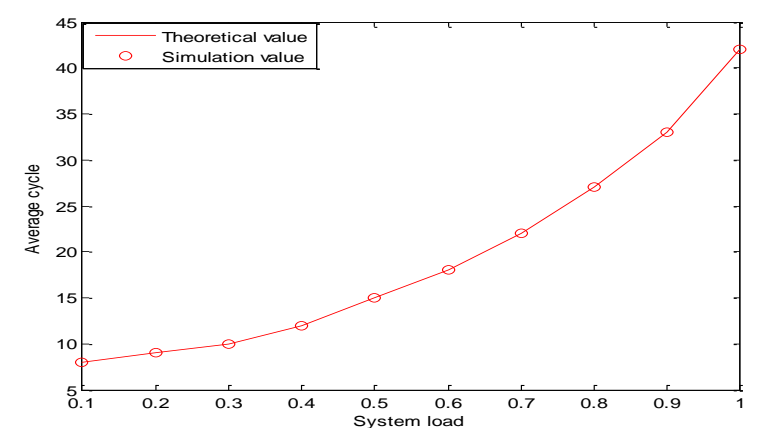

(a) The impact of the system load on mean cycle

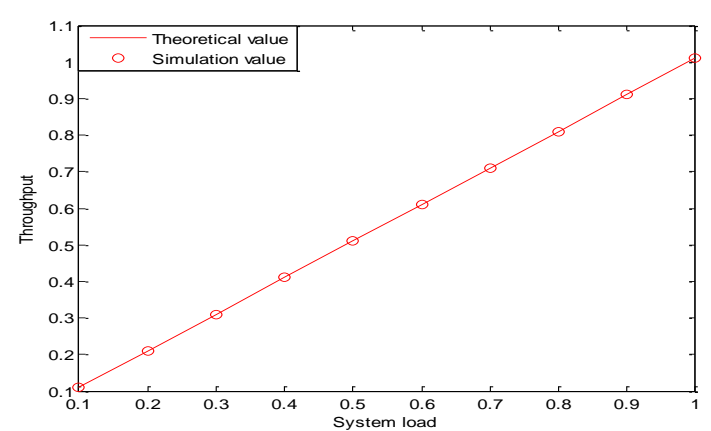

(b) The impact of the system load on the throughput Figure 4. Mean cycle and the throughput

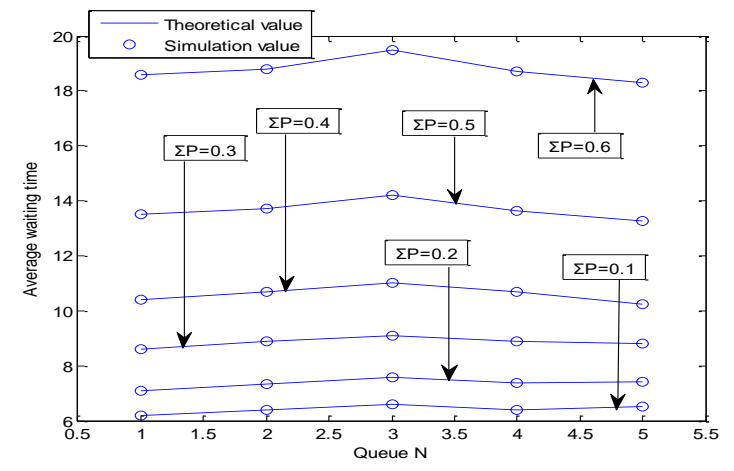

(a) Mean delay of normal queue

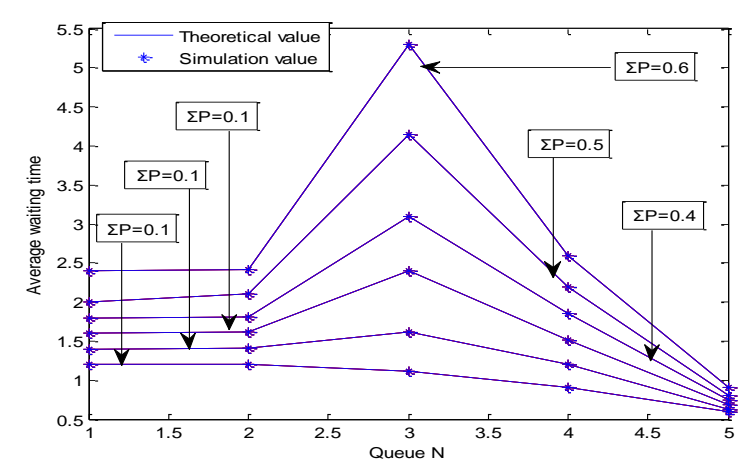

(b) Mean delay of center queue Figure 5. The impact of the system load on mean delay $\left(\sum \rho=\sum_{m=1}^{N} \lambda_{m} \beta_{m}+\lambda_{c} \beta_{c}\right)$ 


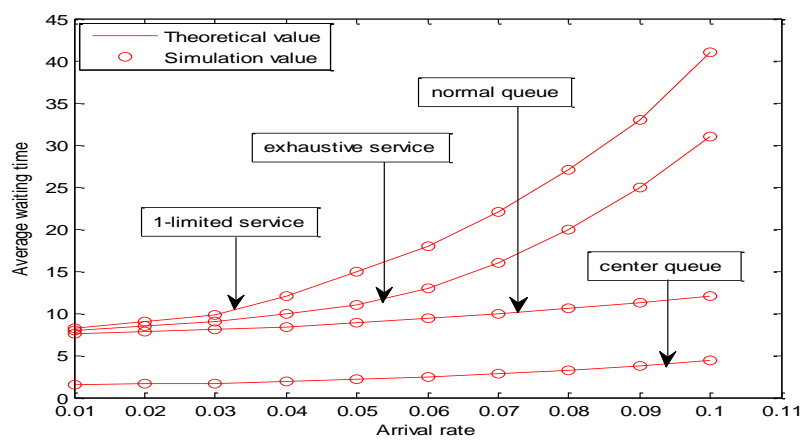

Figure 6. Mean delay of new system compared with the mean delay of a single exhaustive service or single one-limited service

Table 1. Simulation parameters settings of Figures 3 and 4

\begin{tabular}{|c|c|c|c|c|c|}
\hline$i$ & $\lambda_{i}$ & $\lambda_{h}$ & $\gamma_{i}$ & $\beta_{i}$ & $\beta_{h}$ \\
\hline Queue number & Arrival rate & & Switching time & Service time & \\
\hline 1 & 0.001 & 0.01 & 2 & 4 & 1 \\
\hline 2 & 0.006 & 0.01 & 3 & 4 & 1 \\
\hline 3 & 0.003 & 0.01 & 1 & 3 & 1 \\
\hline 4 & 0.04 & 0.01 & 1 & 1 & 1 \\
\hline 5 & 0.01 & 0.01 & 1 & 2 & 1 \\
\hline
\end{tabular}

Table 2. Simulation parameters settings of Figure 5

\begin{tabular}{|c|c|c|c|c|c|}
\hline$i$ & $\lambda_{i}$ & $\lambda_{h}$ & $\gamma_{i}$ & $\beta_{i}$ & $\beta_{h}$ \\
\hline Queue number & Arrival rate & & Switching time & Service time & \\
\hline 1 & 0.002 & 0.01 & 1 & 4 & 1 \\
\hline 2 & 0.005 & 0.01 & 3 & 3 & 1 \\
\hline 3 & 0.003 & 0.01 & 1 & 3 & 1 \\
\hline 4 & 0.04 & 0.01 & 2 & 1 & 1 \\
\hline 5 & 0.01 & 0.01 & 1 & 2 & 1 \\
\hline
\end{tabular}

Table 3. Simulation parameters settings of Figure 6

\begin{tabular}{|c|c|c|c|c|c|}
\hline $\mathrm{N}$ & $\lambda_{i}$ & $\lambda_{h}$ & $\gamma_{i}$ & $\beta_{i}$ & $\beta_{h}$ \\
\hline Queue number & Arrival rate & & Switching time & Service time & \\
\hline 5 & $\{0.001: 0.001: 0.01\}$ & 1 & 1 & 2 & 1 \\
\hline
\end{tabular}

From Figures 3 to 6 , it is clear that the theoretical values and simulation values are identical for the important parameters such as the mean queue length and the mean delay of the system. This shows that the new system is reasonable and efficient.

(1) Figure 3 describes the impact of arrival rate on the mean queue length of the system, which shows that the mean queue length increases as the arrival rate increases. As can be seen from Figure 3, the values of the mean queue length of the center queue are smaller than the values of the mean queue length of the normal queue. It can distinguish the priority of the center queue from the normal queue, because the center queue enjoys higher priority and increased access frequency.

(2) Figures 4(a) and 4(b) respectively describe the mean cycle and throughput increase reasonably with the increase in the system load, and the theoretical values and simulation values are approximately equal.

(3) Figure 5 describes the impact of the system load on mean delay. From this figure, we can see that as the system load increases, the mean delay increases accordingly. As can be seen from the numerical comparison between Figures 5(a) and 5 (b), under the same system load, the values of the mean delay of the center queue are much smaller than the values of the mean delay of the normal queue. The priority of the center queue can be distinguished from that of the normal queue, and network business priorities are clearly differentiated. It also can be seen that Figures 5(a) and 5(b) are non-linear due to the asymmetric system with different parameter settings, which shows that this system is more propitious to multi-access systems in practice.

(4) Figure 6 compares the mean delay of this model with the mean delay of a single exhaustive access policy and limited $(K=1)$ access policy. It can be seen that the mean delays of the normal queue and center queue are less than the mean delay of a single limited $(K=1)$ access policy and exhaustive access policy. This is due to using parallel scheduling 
when switching from the center queue to normal queues, which avoids the switching time and improves the service efficiency of the center queue and normal queue.

\section{Simulation Design of the Mechanism on TinyOS}

The TinyOS operating system is an open source embedded operating system tailored by David Culler of the University of California, Berkeley for wireless sensor networks. It supports a variety of different hardware platforms. Using nesC language and component-based, layered design programming ideas, the code has very high reusability and is suitable for the characteristics of wireless sensor network nodes [22-23].

The above two-level asymmetric polling control system was designed and developed based on TinyOS and CC2538cb as the sensor node. According to system principle, after the normal node in the cluster receives a beacon frame, it sends packets with an asymmetric one-limited $(K=1)$ access policy. After the center node in the cluster receives the beacon frame, it sends packets with an exhaustive access policy. At first, node 2 is selected as the center node, and other member nodes in the same cluster numbered from 3 to 12 are selected as normal nodes. The cluster head node 1 first serves the center node 2 , and after finishing the transmission, it will switch to poll normal node 3. After finishing one-packet transmission, it switches to center node 2 again. The same process happens for the normal nodes. The functions of each node and its corresponding address are shown in Table 4. The experimental parameters are listed in Tables 5 and 6, and Table 7 shows the comparisons between the experimental values and the theoretical calculation values.

Table 4. The function of each node and its corresponding address

\begin{tabular}{|c|c|c|}
\hline Node number & Node address & Node function \\
\hline 1 & fe80::212:6d4c:4f00:1 & Cluster head node \\
\hline 2 & fe $80:: 212: 6 d 4 c: 4 f 00: 2$ & Center node \\
\hline 3 & fe $80:: 212: 6 d 4 c: 4 f 00: 3$ & Normal node \\
\hline 4 & fe $80:: 212: 6 d 4 c: 4 f 00: 4$ & Normal node \\
\hline 5 & fe80::212:6d4c:4fo0:5 & Normal node \\
\hline 6 & $\mathrm{fe} 80:: 212: 6 \mathrm{~d} 4 \mathrm{c}: 4 \mathrm{f} 00: 6$ & Normal node \\
\hline 7 & fe80::212:6d4c:4f00:7 & Normal node \\
\hline 8 & fe80::212:6d4c:4f00:8 & Normal node \\
\hline 9 & fe80::212:6d4c:4f00:9 & Normal node \\
\hline 10 & fe $80:: 212: 6 \mathrm{~d} 4 \mathrm{c}: 4 \mathrm{f} 00: 10$ & Normal node \\
\hline 11 & fe $80:: 212: 6 d 4 c: 4 f 00: 11$ & Normal node \\
\hline 12 & fe $80:: 212: 6 \mathrm{~d} 4 \mathrm{c}: 4 \mathrm{f} 00: 12$ & Normal node \\
\hline
\end{tabular}

Table 5. Experimental parameters settings on TinyOS

\begin{tabular}{cccccc}
\hline $\mathrm{N}$ & $\lambda_{i}$ & $\lambda_{h}$ & $\gamma_{i}$ & $\beta_{i}$ & $\beta_{h}$ \\
\hline Node number & Arrival rate & & Switching time & Service time \\
\hline 1 & 0.001 & 0.01 & 2 & 4 & 4 \\
2 & 0.003 & 0.01 & 3 & 3 & 1 \\
3 & 0.006 & 0.01 & 1 & 2 & 1 \\
4 & 0.01 & 0.01 & 2 & 4 & 1 \\
5 & 0.004 & 0.01 & 2 & 4 & 1 \\
6 & 0.01 & 0.01 & 2 & 2 & 1 \\
7 & 0.005 & 0.01 & 2 & 3 & 1 \\
8 & 0.001 & 0.01 & 3 & 2 & 1 \\
9 & 0.006 & 0.01 & 1 & 2 & 1 \\
10 & 0.02 & & 2 & 1 \\
\hline
\end{tabular}

Table 6. Comparisons of mean queue length on TinyOS

\begin{tabular}{|c|c|c|c|c|}
\hline $\mathrm{N}$ & \multicolumn{2}{|c|}{$g_{i}(i)$} & \multicolumn{2}{c|}{$g_{i h}(h)$} \\
\hline Node & $\begin{array}{c}\text { Theoretical } \\
\text { values }\end{array}$ & $\begin{array}{c}\text { Experimental } \\
\text { values }\end{array}$ & $\begin{array}{c}\text { Theoretical } \\
\text { values }\end{array}$ & $\begin{array}{c}\text { Experimental } \\
\text { values }\end{array}$ \\
\hline 1 & 0.0198 & 0.0190 & 0.0244 & 0.0219 \\
\hline 2 & 0.0593 & 0.0635 & 0.0367 & 0.0353 \\
\hline 3 & 0.1185 & 0.1199 & 0.0122 & 0.0142 \\
\hline 4 & 0.1975 & 0.2011 & 0.0244 & 0.0242 \\
\hline 5 & 0.0790 & 0.0801 & 0.0244 & 0.0235 \\
\hline 6 & 0.1975 & 0.1992 & 0.0244 & 0.0105 \\
\hline 8 & 0.0198 & 0.0251 & 0.0122 & 0.0127 \\
\hline 9 & 0.0988 & 0.1047 & 0.0122 & 0.0140 \\
\hline
\end{tabular}


Table 7. Comparisons of mean waiting time on TinyOS

\begin{tabular}{|c|c|c|c|c|}
\hline $\mathrm{N}$ & \multicolumn{2}{|c|}{$E\left(w_{i}\right)$} & \multicolumn{2}{c|}{$E\left(w_{h}\right)$} \\
\hline Node & $\begin{array}{c}\text { Theoretical } \\
\text { values }\end{array}$ & $\begin{array}{c}\text { Experimental } \\
\text { values }\end{array}$ & $\begin{array}{c}\text { Theoretical } \\
\text { values }\end{array}$ & $\begin{array}{c}\text { Experimental } \\
\text { values }\end{array}$ \\
\hline 1 & 0.0198 & 0.0190 & 0.0244 & 0.0219 \\
\hline 2 & 0.0593 & 0.0635 & 0.0367 & 0.0353 \\
\hline 3 & 0.1185 & 0.1199 & 0.0122 & 0.0142 \\
\hline 4 & 0.1975 & 0.2011 & 0.0244 & 0.0242 \\
\hline 5 & 0.0790 & 0.0801 & 0.0244 & 0.0256 \\
\hline 6 & 0.1975 & 0.1992 & 0.0122 & 0.0105 \\
\hline 8 & 0.0198 & 0.0251 & 0.0122 & 0.0127 \\
\hline 10 & 0.0988 & 0.1047 & 0.0122 & 0.0140 \\
\hline
\end{tabular}

According to Tables 6 and 7, the difference between the theoretical values and the experimental values is very small. This further illustrates that the two-level asymmetric polling control system can be verified on TinyOS and is suitable for the data acquisition system of IoT.

\section{Conclusions}

A two-level asymmetric polling control system was proposed in this paper, in which the arrival time and service time for each queue and the switching times between queues are different. It shows that this model is more suitable and flexible for multi-access systems than symmetric polling systems. Another notable contribution of the paper is that network traffic priorities are clearly differentiated by various service strategies, and each normal queue uses 1-limited service to guarantee system fairness. Using the probability generating function and embedded Markov chain, the system model was established. The difference between the values of simulation and the theoretical values is very small and indicates that the new system can efficiently solve the practical problems of priority in WSNs. The system is also designed and verified based on TinyOS.

\section{Acknowledgements}

This work was supported by the National Science Foundation of China (No. 61461054, 61463051, and 61461053).

\section{References}

1. P. M. Kumar and U. Devi-Gandhi, "A Novel Three-Tier Internet of Things Architecture with Machine Learning Algorithm for Early Detection of Heart Diseases," Computers and Electrical Engineering, Vol. 65, No. 1, pp. 222-235, January 2018

2. Q. L. Han, S. Liang, and H. L. Zhang, "Mobile Cloud Sensing, Big Data, and 5G Networks Make an Intelligent and Smart World," IEEE Network, Vol. 29, No. 2, pp. 40-45, March 2015

3. F. H. Bijarbooneh, W. Du, E. C. H. Ngai, X. Fu, and J. Liu, "Cloud - Assisted Data Fusion and Sensor Selection for Internet of Things," IEEE Internet of Things Journal, Vol. 3, No. 3, pp. 257-268, 2016

4. I. Belhajem, Y. B. Maissa, and A. Tamtaoui, "Improving Vehicle Localization in a Smart City with Low Cost Sensor Networks and Support Vector Machines," Mobile Networks and Applications, Vol. 23, No. 4, pp. 854-863, August 2018

5. X. Luo, D. Zhang, L. T. Yang, J. Liu, X. Chang, and H. Ning, "A Kernel Machine-based Secure Data Sensing and Fusion Scheme in Wireless Sensor Networks for The Cyber-Physical Systems," Future Generation Computer Systems, Vol. 61, pp. 8596, August 2016

6. J. M. Williams, R. Khanna, J. P. Ruiz-Rosero, G. Pisharody, Y. Qian, C. R. Carlson, et al., "Weaving the Wireless Web: Toward a Low-Power, Dense Wireless Sensor Network for the Industrial IoT," IEEE Microwave Magazine, Vol. 18, No. 7, pp. 40-63, November-December 2017

7. N. Kumar and D. P. Vidyarthi, "A Green Routing Algorithm for IoT-Enabled Software Defined Wireless Sensor Network," IEEE Sensors Journal, Vol. 18, No. 22, pp. 9449-9460, November 2018

8. Z. Q. Yang and W. Lv, "Wireless Sensor Network Design for Internet of Things," Digital Communication World, Vol. 3, No. 11, pp. 57-58, 2018

9. K. J. Zhang, Q. L. Han, and Z. P. Cai, "RiPPAS: A Ring-based Privacy-Preserving Aggregation Scheme in Wireless Sensor Networks," Sensors, Vol. 17, No. 2, February 2017

10. C. T. Yang, S. T. Chen, J. C. Liu, Y. W. Su, D. Puthal, and R. Ranjan, "A Predictive Load Balancing Technique for Software Defined Networked Cloud Services," Computing, Vol. 101, No. 3, pp. 211-235, March 2019

11. B. B. Li, Y. He, W. Y. Liu, and L. Wang, "Towards Time-Efficient Localized Polling for Large-Scale RFID Systems," Computer Networks, Vol. 150, No. 26, pp. 250-262, February 2019

12. V. Saranya, S. Shankar, and G. R. Kanagachidambaresan, "Energy Efficient Data Collection Algorithm for Mobile Wireless Sensor Network," Wireless Personal Communications, Vol. 105, No. 1, pp. 219-232, March 2019

13. J. J. Zhao, Z. Q. Gu, L. Xu, Z. H. Li, and X. P. Guan, "A Joint Design Method of Hierarchical Topology Control and Network Resource Allocation for Wireless Sensor Networks,” Acta Automatica Sinica, Vol. 17, No. 41, pp. 646-660, 2015 
14. Z. Guan, D. Zhou, and H. W. Ding, "Parallel Scheduling Two-Stage Polling Control Sensor Network MAC Protocol Analysis," Journal of Intelligent Systems, Vol. 9, No. 7, pp. 438-443, 2014

15. X. J. Xie, "Design and Implementation of MAC Protocol based on TinyOS in Wireless Sensor Networks," Yunnan University, Vol. 6, No. 21, pp. 737-739, 2017

16. L. J. Liu, H. W. Ding, Q. L. Liu, and Z. G. Liu, "Design of Polling Access Control Protocol in WSN based on FPGA," Journal on Communications, Vol. 37, No. 10, pp. 181-187, 2016

17. Z. J. Yang, Y. Su, and H. W. Ding, "Analysis of Two-Level Polling System Characteristics of Exhaustive Service and Asymmetrically Gated Service," Acta Automatica Sinica, Vol. 44, No. 12, pp. 2228-2237, 2018

18. Z. G. Liang, "Research on Discrete-Time Exhaustive Service Two-Level Polling System Theory," Yunnan University, Vol. 41, No. 12, pp. 13-18, 2010

19. L. Y. Bao, "Continuous Time Priority and Research on a Limited Service Polling System," Yunnan University, Vol. 43, No. 15, pp. 23-28, 2009

20. M. Yu and Z. Y. Hu, "Higher Order Markov Random Field and Its Application in Scene Understanding," Journal of Automation, Vol. 4, No. 25, pp. 1213-1234, 2015

21. M. A. Boon, I. J. Adan, and O. J. Boxma, "A Two-Queue Polling Model with Two Priority Levels in the First Queue," Discrete Event Dynamic Systems, Vol. 4, No. 20, pp. 511-536, 2010

22. A. Muhammad, S. Muhammad, A. Muhammad-Khalil, and K. Sung-Won, "TinyOS-New Trends, Comparative Views, and Supported Sensing Applications: A Review,” IEEE Sensors Journal, Vol. 16, No. 9, pp. 2865-2889, May 2016

23. S. Galmés and S. Escolar, "Analytical Model for the Duty Cycle in Solar-based EH-WSN for Environmental Monitoring," Sensors, Vol. 18, No. 8, August 2018

Zhijun Yang received his Ph.D. from the School of Information Science and Engineering at Yunnan University. He currently works for the Yunnan Education Department. His research interests include information systems, network and communication engineering, and random multiple access communication systems.

Zheng Liu is a master's student in the School of Information Science and Technology at Yunnan University. His research interests include wireless sensor networks.

Yangyang Sun is a master's student in the School of Information Science and Technology at Yunnan University. His research interests include wireless sensor networks.

Hongwei Ding is a professor at Yunnan University. His research interests include communication and information systems, network and communication engineering, polling multiple access communication theory, and random multiple access communication systems. 